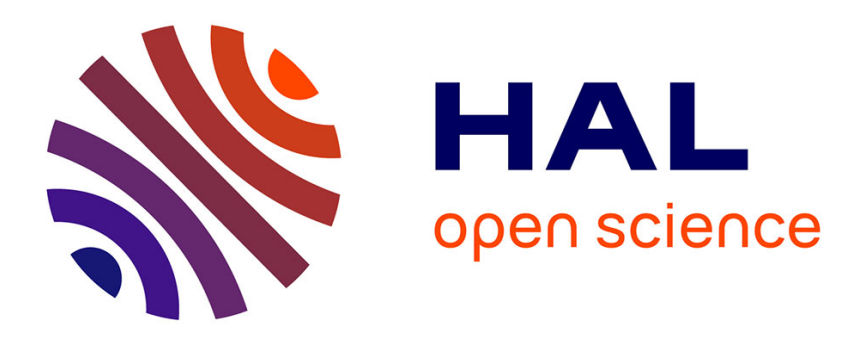

\title{
Mobility of lattice defects: discrete and continuum approaches
}

Olga Kresse, Lev Truskinovsky

\section{To cite this version:}

Olga Kresse, Lev Truskinovsky. Mobility of lattice defects: discrete and continuum approaches. Journal of the Mechanics and Physics of Solids, 2003, 51, pp.1305-1332. 10.1016/S0022-5096(03)00019-X . hal-00111375

\section{HAL Id: hal-00111375 \\ https://hal.science/hal-00111375}

Submitted on 29 Jul 2019

HAL is a multi-disciplinary open access archive for the deposit and dissemination of scientific research documents, whether they are published or not. The documents may come from teaching and research institutions in France or abroad, or from public or private research centers.
L'archive ouverte pluridisciplinaire HAL, est destinée au dépôt et à la diffusion de documents scientifiques de niveau recherche, publiés ou non, émanant des établissements d'enseignement et de recherche français ou étrangers, des laboratoires publics ou privés. 


\title{
Mobility of lattice defects: discrete and continuum approaches
}

\author{
O. Kresse ${ }^{\mathrm{a}}$, L. Truskinovsky $\mathrm{b}^{\mathrm{b}}$, \\ ${ }^{a}$ Department of Aerospace Engineering and Mechanics, University of Minnesota, \\ Minneapolis, MN 55455, USA \\ ${ }^{\mathrm{b}}$ Laboratoire de Méchanique des Solids, UMR 7649, Ecole Polytechnique 91128 Palaiseau, France
}

In this paper, we study a highly idealized model of a moving lattice defect allowing for an explicit, "first principles" computation of a functional relation between the macroscopic config-urational force and the velocity of the defect. The discrete model is purely conservative and contains information only about elasticities of the constitutive elements. The apparent dissipation is due to the presence of microinstabilities and the nonlinearity-induced tunneling of the energy from long to short wavelengths. This type of "radiative damping" is believed to be generic and accounting for a considerable fraction of inelastic irreversibility associated with fracture, plastic-ity and phase transitions. The paper contains direct comparison of the exact lattice solution with various continuum and quasicontinuum approximations. Despite its simplicity, the model can be used directly for the description of dynamic phase transitions in thin films.

Keywords: A. Phase transformations; Defects; B. Dynamics; Lattice model; C. Kinetic relations; Pinning

\section{Introduction}

Irreversible processes in solids can usually be traced to the motion of various lattice defects. Typically, the migration of these defects is a dissipative process with the energy loss in the core region manifesting itself through the localized increase of temperature. In the classical continuum elasticity the drag forces restraining the motion of the defects are absent and they can propagate freely and uniformly with arbitrary velocity. This "freedom" has long been realized as a source of nonuniqueness in the mathematical

\footnotetext{
${ }^{*}$ Corresponding author.

E-mail addresses: omartyn@aem.umn.edu (O. Kresse), trusk@lms.polytechnique.fr (L. Truskinovsky).
} 
structure of the theory, pointing to the necessity of additional constitutive modeling. After the pioneering insights of Eshelby and subsequent developments in the theories of fracture, plasticity and phase transitions it has became common to present the missing constitutive information in the form of a functional relation between the velocity of the defect and the conjugate energy release rate known in different contexts as driving or configurational force.

The function relating velocity of a defect with the corresponding configurational force (kinetic relation) is usually either postulated or obtained from direct experimental measurements. Both approaches bring into constitutive theory a set of parameters of often unclear physical nature. Thus, the most popular phenomenological kinetic relations simply assert that the dissipation is either a quadratic function of velocity or a homogeneous function of degree one. While some of these phenomenological continuum models provide reasonably good overall description, the details of the dissipation process can only be understood by studying the phenomena taking place at the microscopic level, where the discrete nature of the material cannot be neglected. The term discreteness here should be understood rather broadly as inferring to different scales from crystal lattice to grain structure.

In this paper, we investigate the consequences of the direct lattice modeling of a mobile crystal defect and compare the resulting picture with the one provided by various continuum and quasicontinuum approximations. To make the analysis completely transparent we do not attempt to model a particular defect but instead consider a prototypical model. The main requirement to such a model is that elastic energy at the microlevel allows for internal buckling; the associated microscopic instability may then be of quite different nature as suggested, for instance, by the phenomena of twinning or fracture. In the situation when no dissipation is assumed at the microlevel, the macromodel can still be dissipative if the nonlinearity is sufficiently strong to support an irreversible energy flux from macro- to microscales and if the dispersion is sufficiently rich to make the process of energy transmission between the scales possible. The associated "radiative damping" explains at least some of the drag experienced by dislocations, cracks and phase boundaries moving in discrete lattices. A review of the role of radiative damping in plasticity can be found in Al'shitz and Indenbom (1975). A systematic study of the radiative damping (or wave resistance) in fracture mechanics was initiated by the pioneering paper of Slepyan (1981).

Our model essentially reduces to an array of linearly coupled bistable elements (Fig. 1). This mechanical system, a simplified version of which was considered by Slepyan (2000), can be viewed as describing a thin film undergoing "thickness" phase transition or as a model of a side motion of a step on the surface of a generic domain boundary. To drive the system we apply to the elements an external force which creates a bias towards one of the energy wells and makes the propagation of a longitudinal switching wave (kink), energetically favorable. Our goal then is to compute the energy release rate and relate it to the velocity of the kink through a kinetic relation.

This prototypical system represents a two-well adaptation of the discrete FK model (Frenkel and Kontorova, 1938). In its original (purely longitudinal and spatially periodic) form the model was used repeatedly for the description of dynamic dislocations (see Braun and Kivshar, 1998 for a recent review). Following the work of Atkinson 


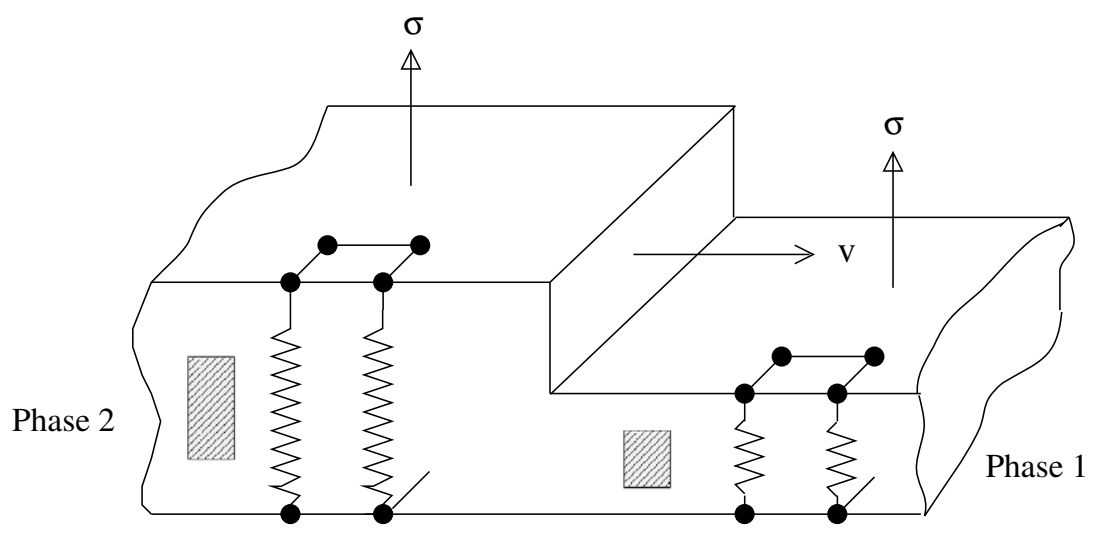

Fig. 1. Schematic configuration of particles around the core of a defect moving with the velocity $v$.

and Cabrera (1965), we chose the nonlinear potential to be two-parabolic with equal elastic moduli. The advantage of this "symmetric" piece-wise linear approximation is that it lends itself to fully analytical treatment through a direct Fourier transform. Closely related discrete piece-wise linear models in fracture mechanics, requiring for their analysis somewhat less transparent Wiener-Hopf technique, have been considered previously by Slepyan (1981, 1982, 2001 a, c) and Marder and Gross (1995). A different model of a phase transition in a chain of bistable springs with "nonsymmetric" energy well structure, which also requires application of the Wiener-Hopf method, was studied by Slepyan and Troyankina (1984), and Slepyan (2001b). In the context of reaction diffusion problems similar piece-wise linear models have been recently studied by Cahn et al. (1999).

In the present paper, we focus on the comparison of the radiation pattern emerging from the discrete model of Atkinson and Cabrera (1965) with the patterns produced by various continuum and quasicontinuum approximations. We recall that in the classical continuum limit subsonic kinks can propagate without any damping: the disappearance of radiative damping in this case is a consequence of a lack of dispersion. To improve the classical model, we consider two different dispersive approximations, polynomial (Boussinesq, 1872) and rational (Rosenau, 1986). Both models augment the classical continuum theory by bringing nonzero internal scales: in the Boussinesq model additional strain gradient terms are added to the free energy, while in the Rosenau model it is the kinetic energy which is modified through the introduction of "transversal inertia." We remark that similar Boussinesq approximation of the FK model has been recently considered by Abeyaratne and Vedantam (1999); a related but different model of the Rosenau type was studied by Theil and Levitas (2000).

As we show in this paper both Boussinesq and Rosenau quasicontinuum approximations produce simple analytic kinetic relations which are in good quantitative agreement with the discrete theory for near sonic and supersonic velocities, where the number of radiated waves in the wake of the defect is small. At small velocities, when the number 
of radiated waves becomes large and the generated waves have lengths comparable to the lattice spacing, the agreement is expectedly poor. In particular, while both quasicontinuum models predict a nonzero trapping domain, the values of the Peierls stress in the approximate models are significantly lower than in the discrete model. Overall, since the Boussinesq model has a very limited domain of application, our analysis suggests that preference should be given to the Rosenau model and the corresponding higher order quasicontinuum approximations.

The paper is organized as follows. In Section 2, we introduce the original discrete model, nondimensionalize variables and formulate the boundary-value problem for the traveling waves. In Section 3, we use Fourier transform to obtain the exact solution of the discrete model. As a part of the solution, we explicitly construct the spectrum of generated waves. In Section 4, we compute the total microscopic energy flux at infinity and relate it to the rate of dissipation at the macroscopic level. This gives a simple analytic expression for the configurational force as a function of the velocity of the defect. Various continuum and quasicontinuum approximations are juxtaposed in Section 5; in each case we obtain a dispersion spectrum and explicitly compute the corresponding kinetic relation. In Section 6 we summarize our results. For convenience, some technical derivations are presented in Appendices A and B.

\section{The model}

Consider an array of particles attached at equal distances $\varepsilon$ to a rigid background by bistable springs with energy density $w\left(\tilde{u}_{n}\right)$. By $\tilde{u}_{n}$ we denote the vertical displacement of the particle with index $n$. To bias one of the energy wells, we introduce an external force per unit length $\tilde{\sigma}$, acting in the direction of the displacement $\tilde{u}_{n}$ and independent of the position of the particle. Finally, we assume that the neighboring particles interact through standard harmonic forces, characterized by an elastic modulus $E$. The potential and kinetic energies of the chain can be written in the form

$$
\mathbf{V}=\sum_{n} \frac{1}{2} E \varepsilon\left(\frac{\tilde{u}_{n+1}-\tilde{u}_{n}}{\varepsilon}\right)^{2}+\varepsilon\left[w\left(\tilde{u}_{n}\right)-\tilde{\sigma} \tilde{u}_{n}\right] ; \quad \mathbf{K}=\sum_{n} \frac{1}{2} \rho \varepsilon \dot{\tilde{u}}_{n}^{2},
$$

where $\rho$ is the mass density per unit spring length. The Euler-Lagrange equations are then

$$
\rho \varepsilon \ddot{\tilde{u}}_{n}-\frac{E}{\varepsilon}\left(\tilde{u}_{n+1}-2 \tilde{u}_{n}+\tilde{u}_{n-1}\right)+\varepsilon\left[w^{\prime}\left(\tilde{u}_{n}\right)-\tilde{\sigma}\right]=0 .
$$

To overcome considerable analytical difficulties associated with solving Eq. (2) for the general $w$, we consider a special double-well potential represented by two parabolas (Fig. 2)

$$
w\left(\tilde{u}_{n}\right)= \begin{cases}\frac{1}{2} c \tilde{u}_{n}^{2}, & \tilde{u}_{n}<\frac{a}{2}, \\ \frac{1}{2} c\left(\tilde{u}_{n}-a\right)^{2}, & \tilde{u}_{n}>\frac{a}{2}\end{cases}
$$

and study a special class of steady-state motions of the chain given by the traveling wave ansatz $\tilde{u}_{n}(t)=\tilde{u}(\varepsilon n-\tilde{v} t)$. The associated particle motion is relatively simple as 


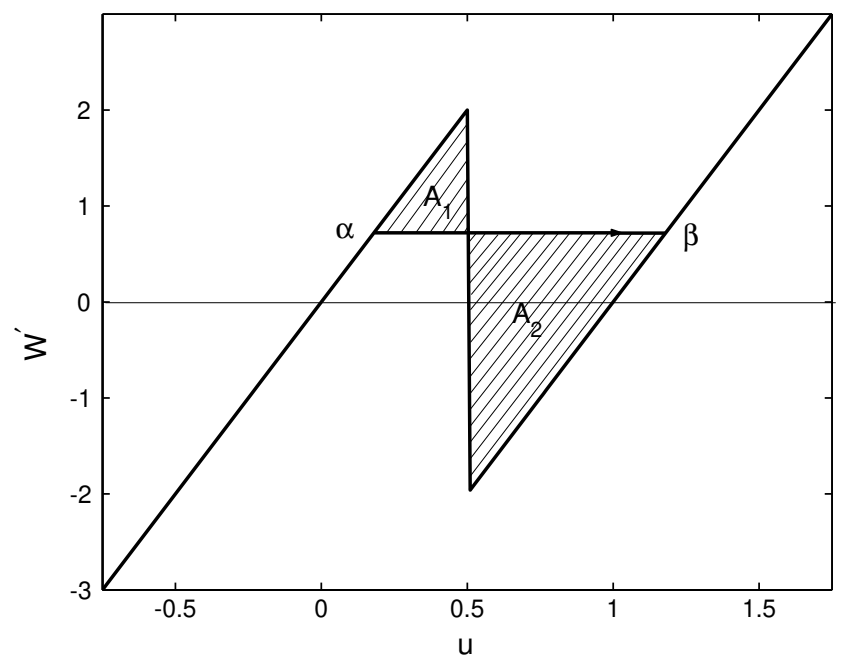

Fig. 2. The piece-wise linear force-elongation relation for the bistable spring (3) with $a=1$ and $c=4$. The propagating defect corresponds to a transition between points $\alpha$ and $\beta$. The configurational force is proportional to the difference in areas $A_{2}-A_{1}$.

the configuration of the chain reproduces itself in discrete moments of time. Without loss of generality we may assume that $\tilde{u}(0)=a / 2$. Then in coordinate system $\tilde{x}=\varepsilon n-\tilde{v} t$ moving with the velocity $\tilde{v}$, all springs located at $\tilde{x}>0$ are in one of the energy wells (phase 1) while all springs located at $\tilde{x}<0$ are in the other energy well (phase 2). By focusing on this particular class of solutions we eliminate the possibility of repeated transition between the wells and restrict our attention to isolated defects. Eq. (2) can then be rewritten in the form

$$
\rho \varepsilon \tilde{v}^{2} \frac{\mathrm{d}^{2} \tilde{u}}{\mathrm{~d} \tilde{x}^{2}}-\frac{E}{\varepsilon}[\tilde{u}(\tilde{x}+\varepsilon)-2 \tilde{u}(\tilde{x})+\tilde{u}(\tilde{x}-\varepsilon)]+\varepsilon[c \tilde{u}(\tilde{x})-\tilde{\sigma}-a c H(-\tilde{x})]=0,
$$

where $H$ is the Heaviside function. As we see the nonlinearity was replaced by an additional force of known magnitude added at each bond that has snapped due to the advancement of the defect.

To highlight the essential parameters, it is convenient to use nondimensional variables

$$
x=\frac{\tilde{x}}{\varepsilon}, \quad u=\frac{\tilde{u}}{a}, \quad v=\tilde{v} \sqrt{\frac{\rho}{E}}, \quad \sigma=\frac{\tilde{\sigma}}{a c} .
$$

In the dimensionless form the nonlinear differential advance-delay equation (4) can be rewritten as

$$
v^{2} \frac{\mathrm{d}^{2} u}{\mathrm{~d} x^{2}}-[u(x+1)-2 u(x)+u(x-1)]+\Omega_{0}^{2}[u(x)-\sigma-H(-x)]=0,
$$

where we introduced the main nondimensional parameter of the problem

$$
\Omega_{0}=\varepsilon \sqrt{\frac{c}{E}} .
$$


For consistency of the model we must require that

$$
u(0)=\frac{1}{2}
$$

and

$$
u(x)<\frac{1}{2}, \quad x>0 ; \quad u(x)>\frac{1}{2}, \quad x<0 .
$$

The last two inequalities oblige the solution to stay inside the two designated energy wells and play the role of the admissibility conditions.

Observe that configurations of the chain at $x= \pm \infty$ must correspond to static equilibrium. By selecting the two limiting states in different energy wells we obtain the following boundary conditions:

$$
u(x) \rightarrow \begin{cases}\sigma, & x \rightarrow \infty \\ \sigma+1, & x \rightarrow-\infty\end{cases}
$$

Since we expect a nonzero radiation of elastic waves at $\pm \infty$ these boundary conditions should be understood as describing only the average values of the displacement fields. The boundary conditions (10) are compatible with admissibility conditions if $\sigma<\sigma_{0}=$ $\frac{1}{2}$, which represents the ultimate (spinodal) strength of our nonlinear springs.

\section{Solution of the discrete problem}

The piece-wise linear discrete boundary-value problem (6)-(10), can be solved by the complex Fourier transform. We leave the details to Appendix A where we extended the methodology introduced in the dislocation context by Atkinson and Cabrera (1965). The solution can be written as a combination of plane waves

$$
u(x)= \begin{cases}\sigma-\Omega_{0}^{2} \sum_{k \in M_{+}} \frac{\mathrm{e}^{\mathrm{i} k x}}{k L^{\prime}(k)}, & x>0 ; \\ \sigma+1+\Omega_{0}^{2} \sum_{k \in M_{-}} \frac{\mathrm{e}^{\mathrm{i} k x}}{k L^{\prime}(k)}, & x<0 .\end{cases}
$$

Here

$$
L(k)=\Omega_{0}^{2}+4 \sin ^{2} \frac{k}{2}-v^{2} k^{2}
$$

and the participating wave numbers, characterizing linear modes generated ahead and behind the moving kink, are arranged in sets $M_{+}$and $M_{-}$. We recall that a plane wave $\mathrm{e}^{\mathrm{i}(k n-\Omega t)}$ can be a part of the solution if and only if its frequency satisfies the dispersion relation for the linear problem inside the wells $\Omega^{2}=\Omega_{0}^{2}+4 \sin ^{2} k / 2$. The same plane wave will also be a part of the traveling wave solution if $\Omega(k)=v k$ which means that phase velocity is equal to the velocity of the traveling wave. By applying these two conditions we obtain that the wave numbers constituting the sets $M_{+}$and $M_{-}$must 


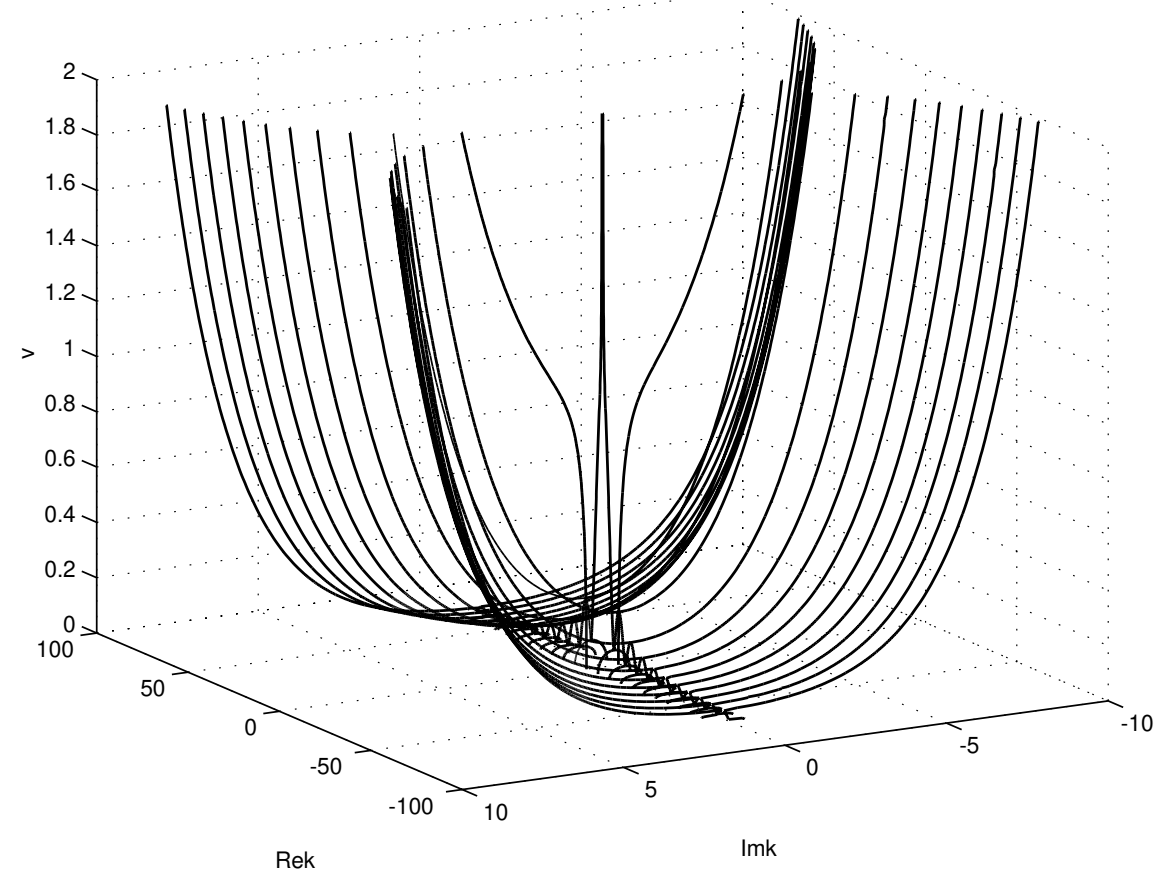

Fig. 3. A complete set of wave numbers generated by the kink moving with velocity $v$ (solutions of the equation $L(k)=0$ at $\left.\Omega_{0}=0.5\right)$.

necessary satisfy the condition

$$
L(k)=0,
$$

which, of course, also follows from the inversion of the Fourier transform (see Appendix A).

At a given value of $v$ Eq. (13) may have real and complex roots $k$ (Fig. 3). An infinite number of complex roots correspond to exponentially damped localized modes describing the core region. The complex roots appear in symmetric quadruples; among them there is always a couple of symmetric pure imaginary roots providing a monotone contribution. An additional finite number of real roots represent acoustic radiation emitted by the defect. These modes must satisfy radiation conditions: for the radiative waves ahead of the moving kink the group velocity must be larger than the phase velocity, while for the waves behind the kink, the group velocity must be smaller than the phase velocity. From the definition of the group velocity we obtain $v_{\mathrm{g}}=\Omega^{\prime}(k)=v+L^{\prime}(k) / 2 v k$.

Now we are in the position to specify the sets $M_{+}$and $M_{-}$

$$
M_{ \pm}=\left\{k: L(k)=0 ;\{\operatorname{Im} k \gtrless 0\} \cup\left\{\operatorname{Im} k=0, k L^{\prime}(k) \gtrless 0\right\}\right\} .
$$

The radiative modes with real wave numbers are illustrated in Fig. 4 (curve D). One can see that for every value of $v$ there exists an odd number of real roots $k$, describing symmetric couples of emitted waves ahead and behind the kink, and an additional 


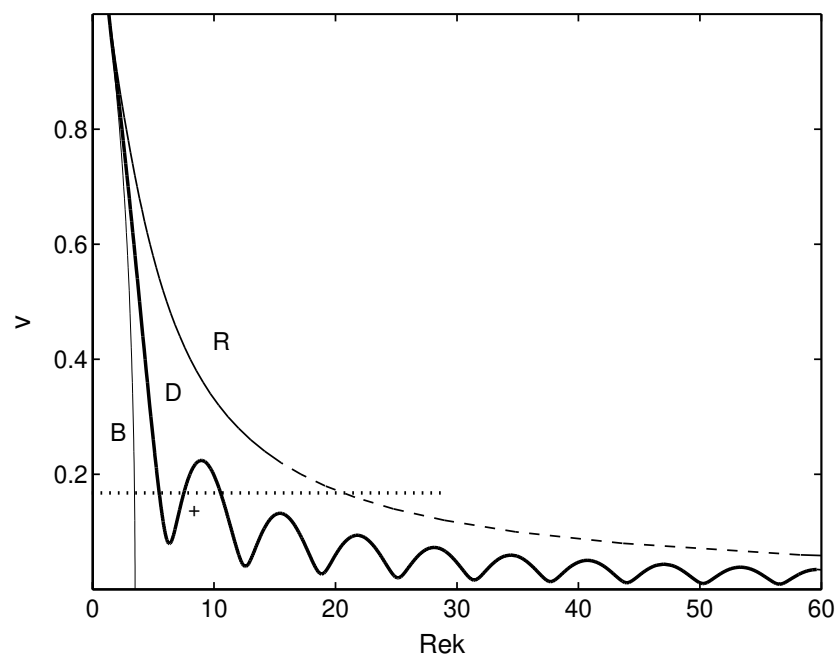

Fig. 4. Propagating waves with the real wave numbers radiated by a kink moving with velocity $v$. Waves marked by "+" and "-" propagate ahead and behind the front, respectively. The three different curves correspond to a discrete (D) and to the two quasicontinuum models (B) and (R) (see the text). Parameters are the same as in Fig. 3.

wave in the wake. We also observe, that whenever $L^{\prime}(k)=0$, two distinct radiative modes merge and for these special values of $v$ the group velocity of a radiated wave is equal to its phase velocity. The amplitudes of the corresponding resonant modes diverge, and the solution blows up because the energy cannot escape from the core region. One can show that the appearance of resonances is not generic and is related to the over-symmetric setting of the model.

After the set of excited modes is known one can use the explicit relations (11) to construct the profiles of the displacement field. The associated $\sigma$ required to sustain the steady motion of the defect can be found from the matching condition (8) (see Appendix A). Several representative profiles of the displacement field for different values of $v$ are shown in Fig. 5. Notice that in the interval $v>0.2243$ the oscillations can be seen only in the wake of the defect, which is in agreement with the imposed radiation conditions: in this interval the only intersection of the line $\Omega=v k$ with the curve $\Omega=\Omega(k)$ satisfies $k L^{\prime}(k)<0$ which means that the group velocity is smaller than the phase velocity. The picture changes as we cross the resonance velocity $v=0.2243$ because now the oscillations are present on both sides of the moving front. For instance, in the interval $0.1324<v<0.2243 \mathrm{Eq}$. (13) has three real roots, responsible for one propagating mode ahead $\left(k L^{\prime}(k)>0\right)$ and two propagating modes behind $\left(k L^{\prime}(k)<0\right)$ the defect.

From Fig. 5(c,d) one can see that the admissibility conditions (9) may not be satisfied below the first resonance velocity and therefore the corresponding traveling wave solutions of form (11) do not exist. More systematic analysis shows that most probably the whole velocity interval $v<0.26$ must be excluded (see similar conclusions in 


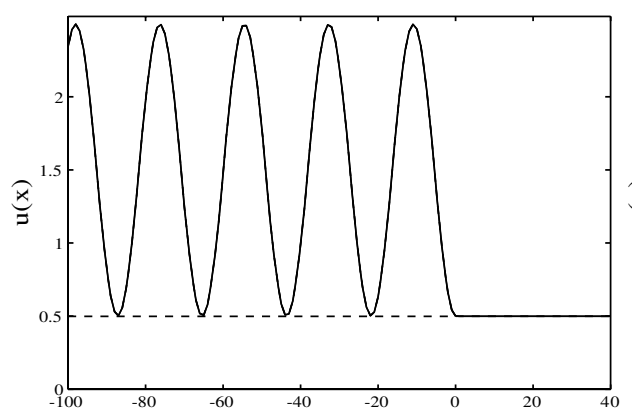

(a)

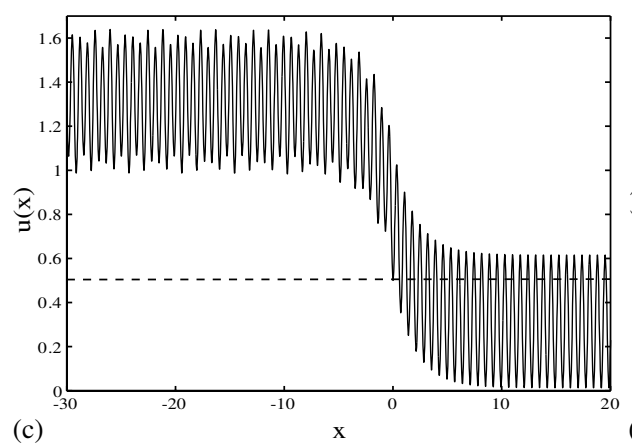

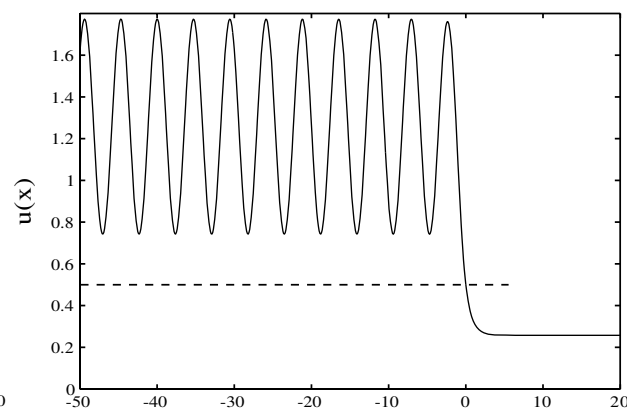

(b)

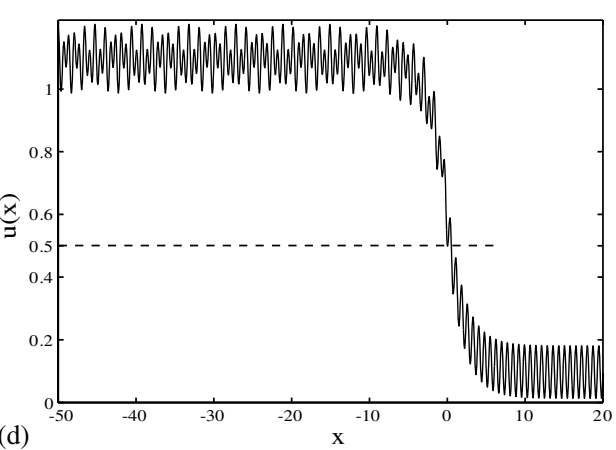

Fig. 5. The displacement field around the core of a moving defect at $v=2.0$ (a); $v=1.0$ (b); $v=0.224$ (c); $v=0.22(\mathrm{~d})$. Here $\Omega_{0}=0.5$.

Marder and Gross, 1995). Since in this excluded velocity range one cannot expect the steady motion of the defect, the description of the actual mode of propagation with prescribed average velocity may require a different ansatz.

\section{Kinetic relation}

As it has already been mentioned in the Introduction, the propagation of a defect through the lattice can be viewed as a succession of internal instabilities associated with the snapping of individual elastic bonds. The inherently dynamic nature of these internal instabilities leads to the generation of high frequency vibrations in the lattice and results in tunneling of the energy from long to short waves. For our special traveling wave solutions the energy transfer between the scales shows as the radiation at infinity. To account for this loss at the macrolevel one can compute the rate of change of the total energy

$$
\frac{\mathrm{d}(\mathbf{V}+\mathbf{K})}{\mathrm{d} t}-\frac{\mathrm{d} \mathbf{A}}{\mathrm{d} t}=\frac{\mathrm{d} \mathbf{Q}}{\mathrm{d} t} .
$$

Here $\mathrm{dA} / \mathrm{d} t$ is the power supplied by the loading device and $\mathrm{d} \mathbf{Q} / \mathrm{d} t$ is the associated "heat" generation. Due to the trivial thermodynamic nature of the system, the function 
$\mathbf{R}(v)=-\mathrm{d} \mathbf{Q} / \mathrm{d} t$ may be interpreted as the rate of dissipation and expressed as a product of the configurational force $G(v)$ and the generalized flux represented in our case by the velocity of the defect $v$

$$
\mathbf{R}(v)=G(v) v .
$$

The relation between the configurational force $G$ and the velocity $v$ will be called kinetic relation. Kinetic relations are routinely employed in the modeling of various lattice defects ranging from twin boundaries to crack tips. Our $G$ can be viewed as an analog of the Peach-Koehler force in the theory of dislocations, of the $\mathrm{J}$ integral in the theory of cracks and of the Eshelby force in the theory of phase transitions.

To obtain an explicit kinetic relation in the present case, we notice that the rate of macroscopic energy loss can be computed as the sum of the microscopic energy fluxes at infinity (e.g. Slepyan, 2001a, b, c). We can write

$$
\mathbf{R}(v)=\mathbf{R}_{+}(v)+\mathbf{R}_{-}(v),
$$

where $\mathbf{R}_{ \pm}(v)$-are the energy fluxes at $\pm \infty$, respectively. To compute $\mathbf{R}_{ \pm}(v)$ we observe that outside the core region the governing equations are linear and therefore the energy flux associated with an individual mode is equal to the product of the averaged density of the total energy $\left\langle\mathbf{G}_{k}\right\rangle$ and the relative velocity of the energy transport with respect to the front $\left|v_{g}-v\right|$. In particular

$$
\begin{aligned}
& \mathbf{R}_{+}(v)=\sum_{k \in N_{+}}\left\langle\mathbf{G}_{k}\right\rangle_{+}\left(v_{g}-v\right), \\
& \mathbf{R}_{-}(v)=\sum_{k \in N_{-}}\left\langle\mathbf{G}_{k}\right\rangle_{-}\left(v-v_{g}\right) .
\end{aligned}
$$

Here $\mathbf{G}_{k}$ represents the sum of kinetic and potential energies per particle carried by a linear wave with the wave number $k$; \langle\rangle denotes averaging over sufficiently large number of periods. In Eq. (18) we have used the fact that due to the asymptotic orthogonality of different modes, the mixed terms do not contribute to the total flux. Since the microscopic radiation involves only propagating modes with real wave numbers, the sets $N_{+}$and $N_{-}$can be defined as the subsets of $M_{+}$and $M_{-}$satisfying additional condition $\operatorname{Im} k=0$.

The average energy density associated with the radiative modes can be computed directly from the definition

$$
\left\langle\mathbf{G}_{k}\right\rangle=\left\langle\mathbf{G}-\mathbf{G}_{0}\right\rangle_{k},
$$

where

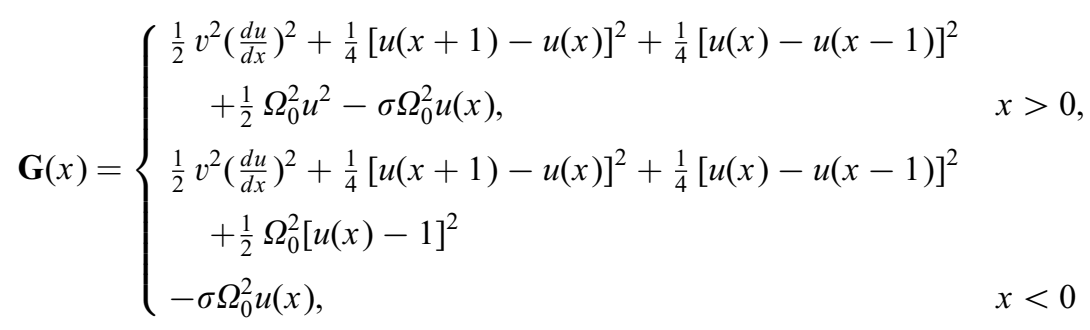


is the total energy per particle and

$$
\mathbf{G}_{0}= \begin{cases}-\frac{1}{2} \Omega_{0}^{2} \sigma^{2}, & x>0, \\ -\frac{1}{2} \Omega_{0}^{2} \sigma^{2}-\Omega_{0}^{2} \sigma, & x<0\end{cases}
$$

is the corresponding energy of the homogeneous states. For an individual mode characterized by the real wave number $k$ we obtain

$$
\begin{aligned}
\left\langle\mathbf{G}_{k}\right\rangle_{ \pm}= & -\frac{\Omega}{2 \pi v} \int_{n}^{n-2 \pi / k}\left[\frac{1}{2} v^{2}\left(\frac{\mathrm{d} u_{k}}{\mathrm{~d} x}\right)^{2}+\frac{1}{4}\left(u_{k}(x+1)-u_{k}(x)\right)^{2}\right. \\
& \left.+\frac{1}{4}\left(u_{k}(x)-u_{k}(x-1)\right)^{2}+\frac{1}{2} \Omega_{0}^{2}\left(u_{k}(x)\right)^{2}\right] \mathrm{d} x \\
= & 2 \frac{\Omega_{0}^{4} v^{2}}{\left[L^{\prime}(k)\right]^{2}} .
\end{aligned}
$$

Now by using Eq. (18) we can rewrite the expressions for the rate of radiative damping in the form

$$
\mathbf{R}(v)=v \Omega_{0}^{4} \sum_{k \in N_{+}} \frac{1}{k L^{\prime}(k)}-v \Omega_{0}^{4} \sum_{k \in N_{-}} \frac{1}{k L^{\prime}(k)} .
$$

Finally, comparing this formula with Eq. (16) we obtain an explicit representation for the configurational force

$$
G=\Omega_{0}^{4} \sum_{k \in N_{ \pm}} \frac{1}{k\left|L^{\prime}(k)\right|} .
$$

The right-hand side of this expression can in principle be computed for any given $v$, which furnishes a desired relation between the configurational force $G$ and velocity $v$.

Before presenting the results of the numerical computations, it is instructive to check the consistency of our approach and compute the expression for configurational force in a different way. Indeed, since the macroscopic motion at $x= \pm \infty$ is absent and the potential energies of the limiting homogeneous configurations are different, a moving defect converts macroscopic energy of the system at a rate

$$
\begin{aligned}
\mathbf{R}(v) & =\left[\mathbf{G}_{0}(+\infty)-\mathbf{G}_{0}(-\infty)\right] v \\
& =\left\{w_{0}(+\infty)-w_{0}(-\infty)-\Omega_{0}^{2} \sigma\left[u_{0}(+\infty)-u_{0}(-\infty)\right]\right\} v \\
& =\Omega_{0}^{2} \sigma v .
\end{aligned}
$$

By comparing the two expressions for $\mathbf{R}$, we obtain the relation between the driving force $G$ and the applied force per unit length $\sigma$

$$
G=\Omega_{0}^{2} \sigma .
$$

This formula can also be interpreted geometrically as the difference between the shaded areas $A_{2}-A_{1}$ in Fig. 2. The fact that the configurational force is a linear function of 
applied stress is not generic; in the case of different moduli of the phases this relation is quadratic. One can also see that due to (A.15) the two ways of computing the configurational force represented by Eqs. (24) and (26) are consistent.

We begin the analysis of the resulting kinetic relation by first investigating two important limiting cases:

1. Fast waves: $v \rightarrow \infty$. According to the dispersion relation in this limit $k \rightarrow 0$ and $k L^{\prime}(k) \rightarrow-2 \Omega_{0}^{2}$, which gives

$$
\lim _{v \rightarrow \infty} G=\frac{\Omega_{0}^{2}}{2} .
$$

One can see that the stress required to maintain the limiting motion approaches asymptotically the value $\sigma=\frac{1}{2}$ which corresponds to the spinodal limit (ultimate strength); as we have already mentioned, no solution is possible for $\sigma>1 / 2$.

2. Slow waves: $v \rightarrow 0$. In this limit, the solution exhibits a growing number of excited lattice waves and because of the accumulation of resonances the asymptotic behavior of $G$ is not straightforward. However, one can show that

$$
\lim _{v \rightarrow 0} G=\Omega_{0}^{2} \sigma_{\mathrm{p}}^{D}
$$

In this expression

$$
\sigma_{\mathrm{p}}^{D}=\frac{\Omega_{0}}{2 \sqrt{4+\Omega_{0}^{2}}}
$$

is exactly the Peierls force (or critical toughness) in the static problem specifying the upper limit of the "lattice trapping" (see Appendix B for details). The consistency of static and dynamic computations confirms that the traveling wave regimes originate exactly from the bifurcation of the static solutions.

In the intermediate range of velocities between $v=0$ and $\infty$ the form of the kinetic relation can be obtained numerically from the precise determination of the sets $N_{ \pm}$at each value of $v$. The results for $\Omega_{0}=0.5$ are presented in Fig. 6. An interesting feature of the obtained kinetic curve is the presence of the infinite number of resonances at low velocities. As we have mentioned in the previous section, the traveling waves in this area do not exist and the corresponding branches should be excluded. Notice also that the kinetic relation can be smoothly extended from subsonic into supersonic region, even though the use of the term "sonic velocity" in the present model is somewhat questionable. The complete analysis of stability of the traveling wave solutions depicted in Fig. 6 presents a considerable challenge.

\section{Quasicontinuum approximations}

Our analysis has been so far focused on the discrete model. When the size of the core of the moving defect is mesoscopic the more natural description is furnished by continuum mechanics with most microscopic degrees of freedom integrated out; a quasicontinuum approximation aims at incorporating the leading effects due to discreteness 


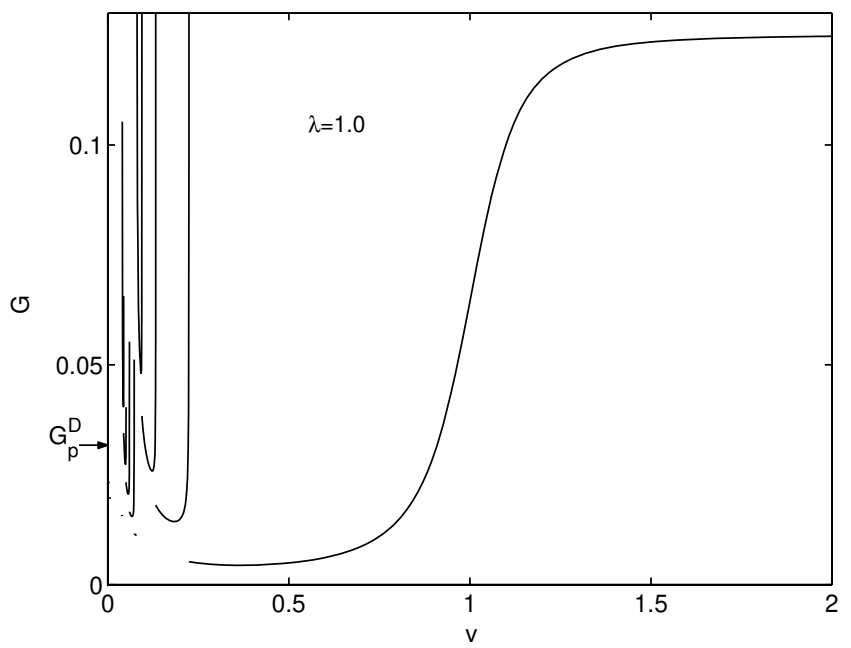

Fig. 6. Configurational force as a function of velocity (kinetic relation) at $\Omega_{0}=0.5$. The low velocity domain around the resonances does not correspond to admissible traveling waves and must be excluded. The corrected picture is shown in Fig. 7.

into the continuum model. Although our discrete model is conservative, the continuum and quasicontinuum approximations may be dissipative since, due to nonlinearity, the energy is not confined at long waves.

To construct continuum and quasicontinuum approximations for our discrete model we replace the advance-delay terms in the main equation (6) by the corresponding higher derivative approximation. We recall that

$$
u(x+1)-2 u(x)+u(x-1)=\left[4 \sinh ^{2}\left(\frac{1}{2} D\right)\right] u(x),
$$

where $D$ is the operator of differentiation. The right-hand side of Eq. (30) can be formally approximated by either polynomial or rational (Pade) expansions of the form (e.g. Wattis, 1993)

$$
4 \sinh ^{2}\left(\frac{1}{2} D\right) \approx D^{2}\left[I+\frac{1}{12}(D)^{2}\right] \approx D^{2}\left[I-\frac{1}{12}(D)^{2}\right]^{-1} .
$$

We begin with the polynomial approximation and rewrite Eq. (6) in the form (see also Abeyaratne and Vedantam, 1999)

$$
u_{x x x x}-12\left(v^{2}-1\right) u_{x x}-12 \Omega_{0}^{2}(u-\sigma)=-12 \Omega_{0}^{2} H(-x) .
$$

As before, the nondimensional displacement field $u(x)$ must satisfy additional admissibility conditions (8)-(9). The nondispersive approximation, obtained by dropping in expansion (32) the terms with spatial derivatives higher than two, represents a classical continuum limit. In this limit Eq. (32) takes the form

$$
\left(v^{2}-1\right) u_{x x}+\Omega_{0}^{2} u=\Omega_{0}^{2}[\sigma+H(-x)] .
$$


By applying the Fourier transform (as in the discrete problem) we obtain

$$
u(x)=\sigma-\frac{\Omega_{0}^{2}}{2 \pi \mathrm{i}} \int_{\gamma} \frac{\mathrm{e}^{\mathrm{i} k x}}{k L_{\mathrm{c}}(k)} \mathrm{d} k,
$$

where now

$$
L_{\mathrm{c}}(k)=\Omega_{0}^{2}+k^{2}\left(1-v^{2}\right) .
$$

In the continuum model the dispersion relation $L_{\mathrm{c}}(k)=0$ has only two roots

$$
k_{1,2}= \pm \sqrt{\frac{\Omega_{0}^{2}}{v^{2}-1}},
$$

which are real if $v>1$ (supersonic region) and pure imaginary if $v<1$ (subsonic region). By using the radiation conditions we can compute the integral in Eq. (34) and obtain in the subsonic case

$$
u(x)= \begin{cases}\sigma+\frac{1}{2} \mathrm{e}^{-x \sqrt{\Omega_{0}^{2} /\left(1-v^{2}\right)}}, & x>0, \\ \sigma+1-\frac{1}{2} \mathrm{e}^{x \sqrt{\Omega_{0}^{2} /\left(1-v^{2}\right)}}, & x<0 .\end{cases}
$$

This classical solution satisfies the admissibility conditions at $\sigma=0$ only. The fact that in the classical theory the defect can propagate at zero force, is not surprising since in this approximation the radiative damping is completely absent.

Now notice that the above subsonic branch of solutions is connected to a branch of sonic "shock waves" at $v=1$. In fact, for $v=1$ the following discontinuous solution can be constructed:

$$
u(x)= \begin{cases}\sigma & \text { if } x>0, \\ \sigma+1 & \text { if } x<0 .\end{cases}
$$

This solution is defined for $0<\sigma<1 / 2$. In the supersonic case, $v>1$, the traveling wave solution satisfying the admissibility conditions takes the form

$$
u(x)= \begin{cases}\frac{1}{2}, & x>0, \\ \frac{3}{2}-\cos \sqrt{\frac{\Omega_{0}^{2}}{v^{2}-1} x,} & x<0 .\end{cases}
$$

This solution, existing only at $\sigma=1 / 2$, describes the motion of a supersonic phase boundary at ultimate stress.

If we now restore the fourth spatial derivative in Eq. (32) we obtain the quasicontinuum Boussinesq model. By applying the Fourier transform we can again represent solution in the form

$$
u(x)=\sigma-\frac{\Omega_{0}^{2}}{2 \pi \mathrm{i}} \int_{\gamma} \frac{\mathrm{e}^{\mathrm{i} k x}}{k L_{\mathrm{B}}(k)} \mathrm{d} k,
$$


where now

$$
L_{\mathrm{B}}(k)=\Omega_{0}^{2}+k^{2}\left(1-v^{2}\right)-\frac{k^{4}}{12} .
$$

In the Boussinesq approximation the dispersion relation $L_{\mathrm{B}}(k)=0$ generates four wave numbers for each value of $v$ : two purely imaginary, describing the structure of the core region and two real, describing the radiation pattern. Explicitly we can write

$$
k_{1,2,3,4}= \pm \sqrt{6\left(1-v^{2}\right)\left[1 \pm \sqrt{1+\frac{\Omega_{0}^{2}}{3\left(1-v^{2}\right)^{2}}}\right]} .
$$

According to radiation conditions we must close the contour $\gamma$ in the upper half-plane for $x>0$ and include one purely imaginary root only; for $x<0$ we must instead close the contour in the lower half-plane and include one purely imaginary root and two real roots. As a result we obtain

$$
u_{ \pm}=\left\{\begin{array}{cc}
\sigma+\frac{1}{4}\left[1+\frac{\sqrt{3}\left(1-v^{2}\right)}{\sqrt{\Omega_{0}^{2}+3\left(v^{2}-1\right)^{2}}}\right] \mathrm{e}^{-l_{ \pm} x}, & x>0 \\
\sigma+1-\frac{1}{4}\left[1+\frac{\sqrt{3}\left(1-v^{2}\right)}{\sqrt{\Omega_{0}^{2}+3\left(v^{2}-1\right)^{2}}}\right] \mathrm{e}^{l_{ \pm} x} & \\
-\frac{1}{2}\left[1 \mp \frac{\sqrt{3}\left(1-v^{2}\right)}{\sqrt{\Omega_{0}^{2}+3\left(v^{2}-1\right)^{2}}}\right] \cos m_{ \pm} x, & x<0
\end{array}\right.
$$

where the choice of signs \pm indicates the cases $v \gtrless 1$, respectively, and where

$$
\begin{aligned}
& l_{ \pm}=\sqrt{ \pm 6\left(v^{2}-1\right)\left[\sqrt{\left.1+\frac{\Omega_{0}^{2}}{3\left(v^{2}-1\right)^{2}} \pm 1\right]}\right.} \\
& m_{ \pm}=\sqrt{ \pm 6\left(v^{2}-1\right)\left[\sqrt{1+\frac{\Omega_{0}^{2}}{3\left(v^{2}-1\right)^{2}}} \mp 1\right]} .
\end{aligned}
$$

Solution (43) satisfies all the admissibility conditions and the corresponding kinetic relation can be obtained in an explicit form (cf. Abeyaratne and Vedantam, 1999)

$$
G=\frac{\Omega_{0}^{2}}{4}\left[1 \pm \sqrt{\frac{3\left(v^{2}-1\right)^{2}}{3\left(v^{2}-1\right)^{2}+\Omega_{0}^{2}}}\right], \quad v \gtrless 1 .
$$




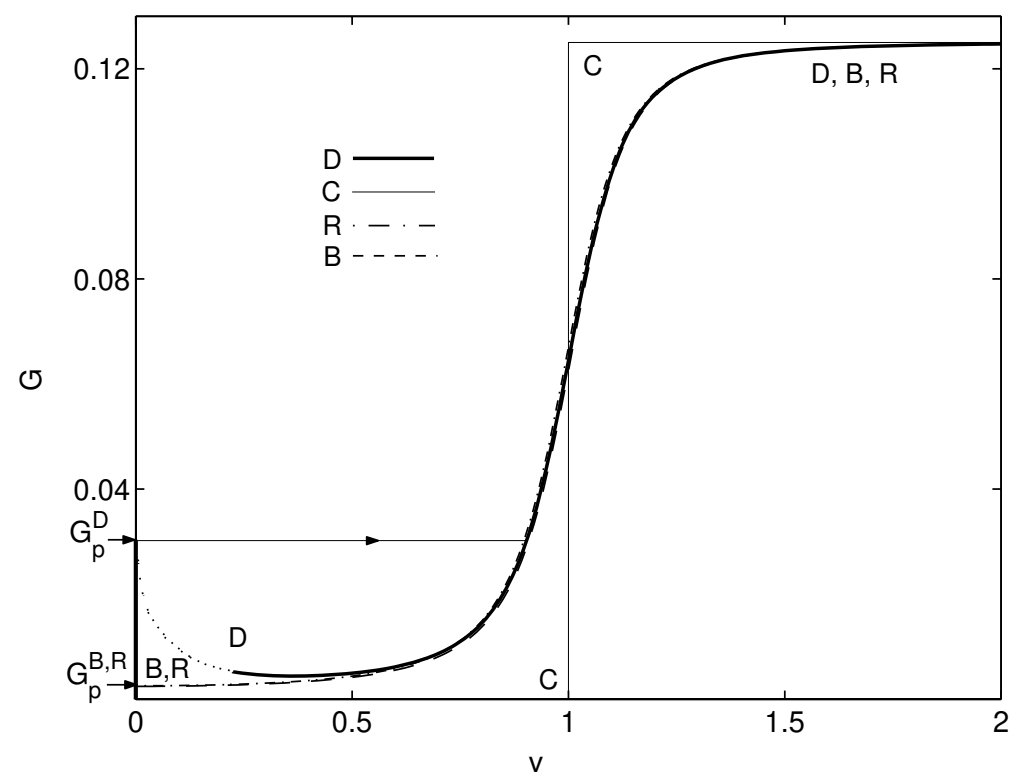

Fig. 7. The configurational force as a function of velocity of the defect in the discrete model (D), continuum model (C) and quasicontinuum models (B) and (R). The horizontal arrow marks the instability limit for the lattice trapping region leading to a transition to a fully developed inertial regime. For all curves $\Omega_{0}=0.5$.

Here if $v \rightarrow \infty$, we obtain the same spinodal limit (27) as in the discrete case. In the other limiting case $v \rightarrow 0$ we obtain

$$
\lim _{v \rightarrow 0} G=\Omega_{0}^{2} \sigma_{\mathrm{p}}^{\mathrm{B}}
$$

where

$$
\sigma_{\mathrm{p}}^{\mathrm{B}}=\frac{1}{4}\left(1-\sqrt{\frac{3}{3+\Omega_{0}^{2}}}\right) .
$$

As we show in Appendix B this value of the applied force matches exactly the static Peierls force computed for the Boussinesq model. The resulting kinetic equation is presented in Fig. 7.

Although the kinetic relation for the traveling waves in the Boussinesq approximation matches the kinetic relation in the discrete problem reasonably well, the initial-value problem for the Boussinesq model is linearly ill-posed with unbounded growth rates at short wavelengths (Pego et al., 1995, Christov et al., 1996). The reason for this failure of the Boussinesq model is that the strain gradient part of the elastic energy in this model is not positive definite (e.g. Kunin, 1982). An alternative (rational) approximation of the delay-advance operator mentioned in Eq. (31), has the same order as the Boussinesq model but has an advantage of always being stable at short waves (Rosenau, 1986). 
In the Rosenau approximation the equation for traveling waves takes the form

$$
\begin{aligned}
& v^{2} D^{4} u-12 D^{2}\left[\left(v^{2}-1-\frac{\Omega_{0}^{2}}{12}\right) u+\frac{\Omega_{0}^{2}}{12} H\left(u-\frac{1}{2}\right)\right]-12 \Omega_{0}^{2}(u-\sigma) \\
& \quad=-12 \Omega_{0}^{2} H\left(u-\frac{1}{2}\right) .
\end{aligned}
$$

For solutions describing an isolated kink, the singular nonlinear equation (48) can be rewritten as two linear equations

$$
v^{2} u_{x x x x}-12\left(v^{2}-1-\frac{\Omega_{0}^{2}}{12}\right) u_{x x}-12 \Omega_{0}^{2}(u-\sigma)= \begin{cases}0, & x>0, \\ -12 \Omega_{0}^{2}, & x<0\end{cases}
$$

supplemented by the following matching conditions at $x=0$ :

$$
[u]=\left[u_{x}\right]=\left[u_{x x x}\right]=0 ; \quad v^{2}\left[u_{x x}\right]=\Omega_{0}^{2} .
$$

Here the square parentheses denote the jump discontinuity []$=()_{+}-()_{-}$. Notice that in the linearized Rosenau model the dispersion equation relating phase velocity of the plane waves to the velocity of the kink and completely characterizing the radiative pattern at infinity, takes the form $L_{\mathrm{R}}(k)=0$, where now

$$
L_{\mathrm{R}}(k)=\Omega_{0}^{2}-\frac{v^{2}}{12} k^{4}+k^{2}\left(1+\frac{\Omega_{0}^{2}}{12}\right)-k^{2} v^{2} .
$$

This equation has four roots: two real and two purely imaginary

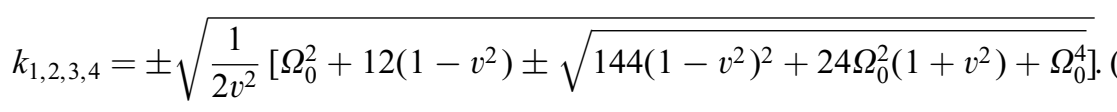

The displacement field representing solution of Eq. (49) and satisfying the matching conditions (50), is given by the formulas

$$
u(x)= \begin{cases}\sigma+\frac{v^{2} k_{1}^{2}-\Omega_{0}^{2}}{2 v^{2}\left(k_{1}^{2}-k_{3}^{2}\right)} \mathrm{e}^{\mathrm{i} k_{3} x}, & x \geqslant 0, \\ \sigma+1-\frac{v^{2} k_{1}^{2}-\Omega_{0}^{2}}{2 v^{2}\left(k_{1}^{2}-k_{3}^{2}\right)} \mathrm{e}^{\mathrm{i} k_{4} x}+\left[\frac{v^{2} k_{1}^{2}-\Omega_{0}^{2}}{2 v^{2}\left(k_{1}^{2}-k_{3}^{2}\right)}-\frac{1}{2}-\sigma\right] & \\ \cos k_{1} x, & x \leqslant 0 .\end{cases}
$$

Here $k_{3}$ and $k_{4}$ indicate purely imaginary roots (52) in the upper and lower half-planes, respectively, and $k_{1}$ is the positive real root. The explicit kinetic relation takes the form

$$
G=\frac{\Omega_{0}^{2}}{2} \frac{\Omega_{0}^{2}-v^{2} k_{3}^{2}}{v^{2}\left(k_{1}^{2}-k_{3}^{2}\right)} .
$$

In the limit $v \rightarrow \infty$ we again obtain condition (27) which agrees with both the discrete and the Boussinesq models. In the alternative limit $v \rightarrow 0$ we obtain

$$
\lim _{v \rightarrow 0} G(v)=\Omega_{0}^{2} \sigma_{\mathrm{p}}^{\mathrm{R}},
$$




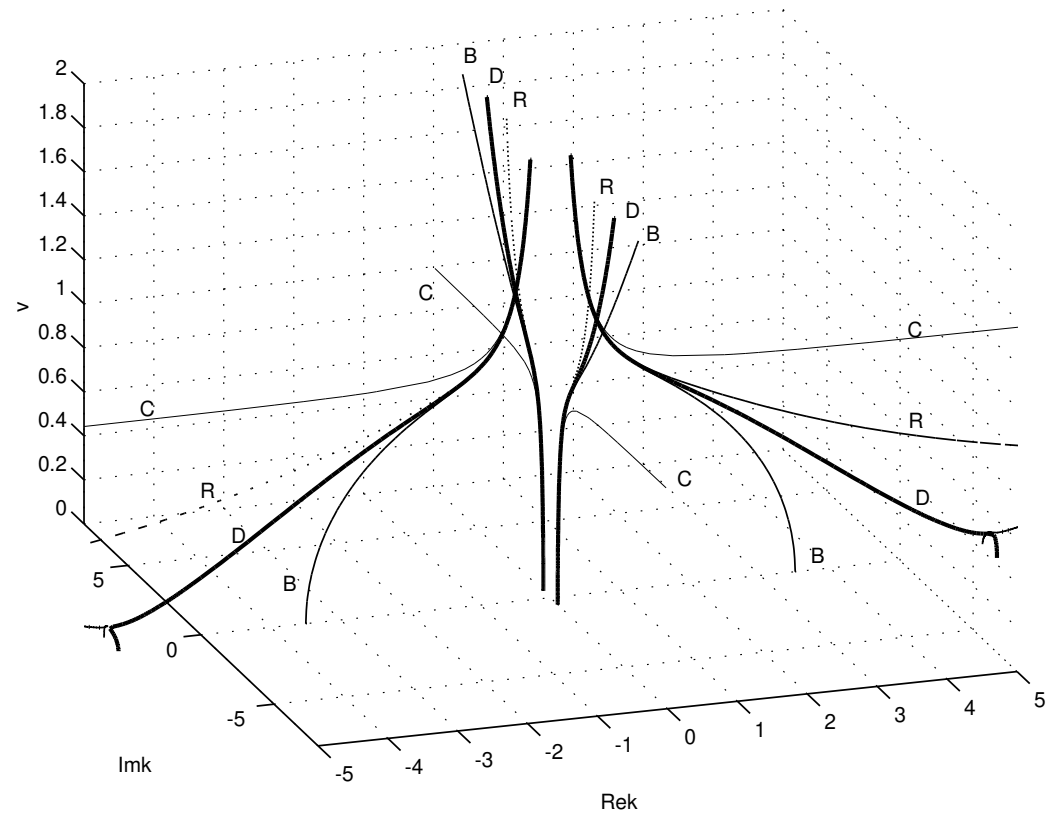

Fig. 8. Comparison of the dispersion spectra for discrete (D), continuum (C), Boussinesq (B) and Rosenau (R) models. Here $\Omega_{0}=0.5$.

where now

$$
\sigma_{\mathrm{p}}^{\mathrm{R}}=\frac{\Omega_{0}^{2}}{2\left(12+\Omega_{0}^{2}\right)} .
$$

To compare all four models we fix the value of the nondimensional parameter $\Omega_{0}$ and superimpose the resulting kinetic relations on one graph (Fig. 7). One can see that the kinetic curves for discrete (D) and both quasicontinuum models (B) and $(\mathrm{R})$ are almost indistinguishable in the region $v>0.2243$ where only one propagating mode is emitted. It is also immediately obvious that the classical continuum model (C) cannot be considered satisfactory since it predicts zero drag everywhere in the subsonic range.

Both dispersive quasicontinuum approximations capture the effects of radiative damping although with different level of detail. To understand the difference between the radiation patterns in different models we present on one graph all four dispersion spectra (Fig. 8). As expected all models essentially agree in the domain of supersonic velocities and long waves where the dispersion spectra in all four theories reduce to two real and two purely imaginary branches. Consequently for $v>1$ solutions generated by all four models are almost identical. At $v<1$ purely imaginary branches in the models (B), (R) and (C) practically coincide with the corresponding branches of the discrete model (D). Therefore, in this velocity range the structure of the nonoscillatory part of 


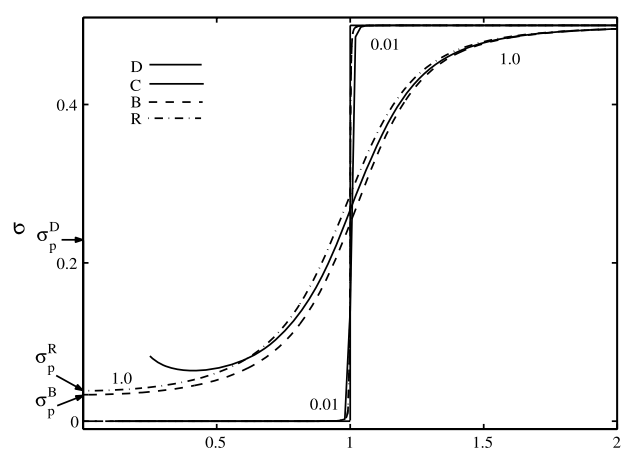

(a)

Fig. 9. (a) Comparison of the discrete model with continuum and quasicontinuum models at different values of $\Omega_{0}$ : force-velocity relations at $\Omega_{0}=0.01$ and $\Omega_{0}=1.0$; (b) the Peierls force for discrete (D), Boussinesq (B) and Rosenau (R) models as a function of $\Omega_{0}$.

the wave field near the core of the defect is similar in all four models. Another general observation is that for real branches, the Boussinesq model provides a lower bound, while the Rosenau model gives an upper bound of the discrete dispersion spectrum (see Fig. 4).

By examining the slopes of the real branches of the dispersion relation in both $(\mathrm{R})$ and (B) models we conclude that they contribute to the wave field only behind the kink. Therefore, these quasicontinuum approximations can be considered appropriate only at $v>0.2243$, because below this resonant velocity the original discrete model exhibits radiation both ahead and behind the kink. At subsonic velocities the Boussinesq model radiates waves with basically one wavelength $(k \approx 3.5)$, while both discrete and Rosenau models develop, as velocity tends to zero, radiation with smaller and smaller wavelengths.

Important conclusions concerning the adequacy of the approximate models can be drawn from the comparison of the static limits for the corresponding kinetic relations. We recall that due to lattice trapping the solutions describing stationary kinks in the discrete model exist in a finite range of applied forces $-\sigma_{\mathrm{p}}^{\mathrm{D}}<\sigma<\sigma_{\mathrm{p}}^{\mathrm{D}}$, where $\sigma_{\mathrm{p}}^{\mathrm{D}}$ is the corresponding Peierls force. In the continuum approximation static solutions exist only at zero stress, which means that $\sigma_{\mathrm{p}}^{\mathrm{C}}=0$. In both Boussinesq and Rosenau approximations the lattice trapping phenomenon is preserved. The relation among the values of Peierls forces $\sigma_{\mathrm{p}}^{\mathrm{D}}, \sigma_{\mathrm{p}}^{\mathrm{B}}$ and $\sigma_{\mathrm{p}}^{\mathrm{R}}$ at different values of $\Omega_{0}$ is illustrated in Fig. 9. As one can see from this figure and from the comparison of the kinetic relations at different values of $\Omega_{0}$, the agreement between the models improves as the reference length of the discrete chain gets smaller or the harmonic coupling between the snap-springs gets stronger $\left(\Omega_{0} \rightarrow 0\right)$.

It is also instructive to compare the displacement fields obtained in different models (see Fig. 10). As expected, in the supersonic and near sonic cases, the displacement field obtained in discrete (D) and in different quasicontinuum models practically coincide (Fig. 10a,b): all models predict exponentially damped displacements at $x>0$ and 


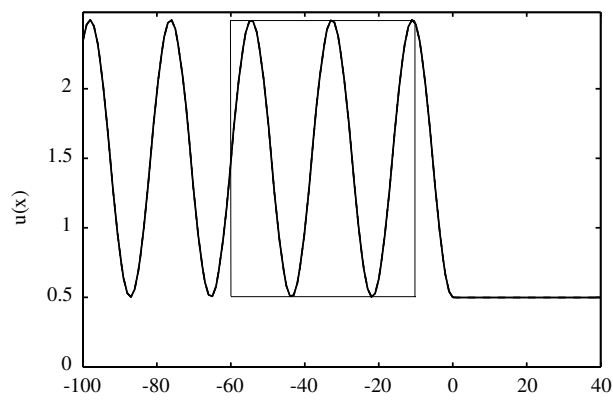

(a)

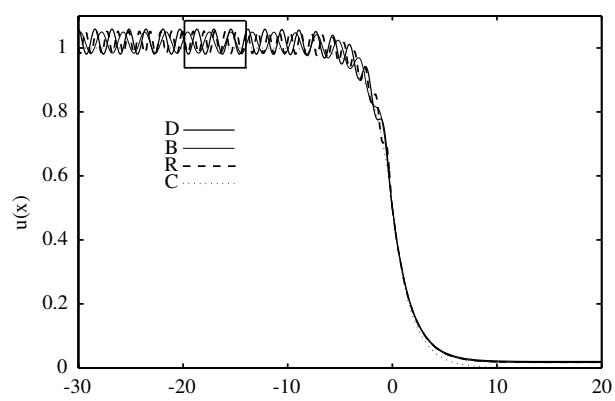

(c)

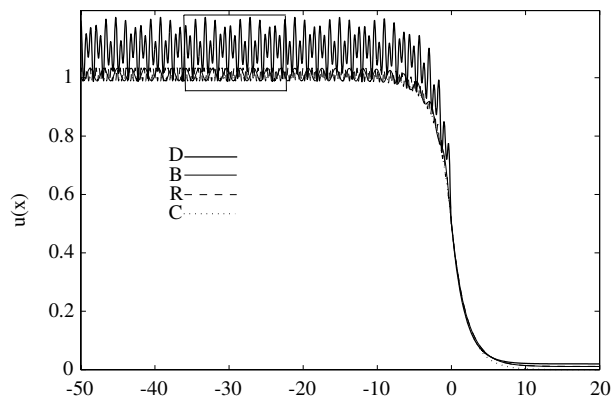

(e)

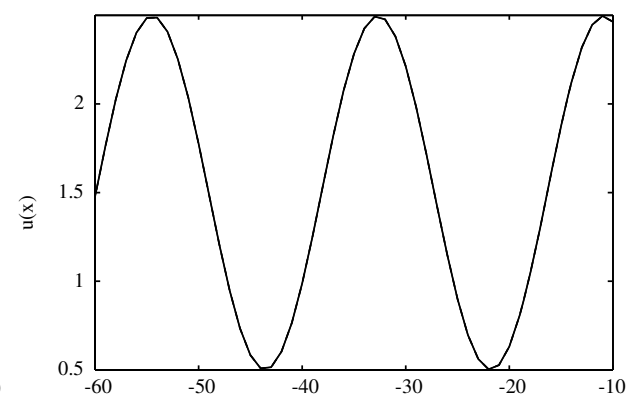

(b)

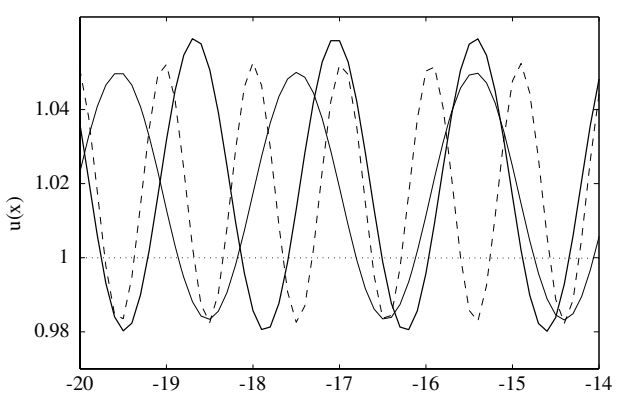

(d)

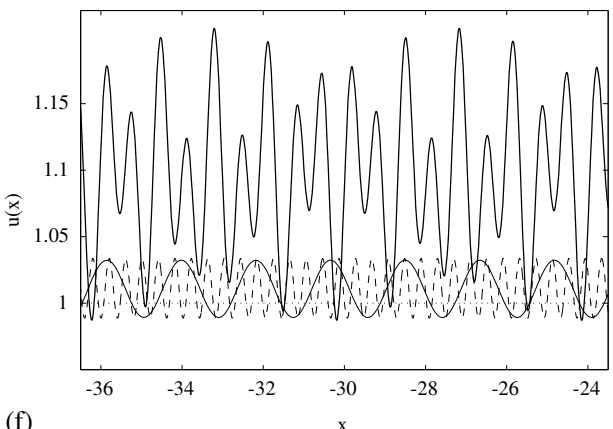

Fig. 10. Displacement fields for the discrete (D), Boussinesq (B), Rosenau (R) and continuum (C) models at $v=2.0$ (a), (b), $v=0.5$ (c), (d) and $v=0.22$ (e), (f). In all graphs $\Omega_{0}=0.5$.

infinite oscillatory tails at $x<0$. The agreement is so good because only long waves are involved. Outside this range the discrete model basically agrees with the quasicontinuum models in the velocity interval bounded below by the largest resonant velocity for the discrete model. Below this critical velocity the discrete model exhibits simultaneous radiation ahead and behind the kink while the quasicontinuum models show oscillatory components only behind the moving defect. The detailed comparison in this range of velocities does not make much sense because no discrete solution seems to be admissible. It is interesting, however, that at extremely small velocities, the behavior of 
the three models is very different: the Boussinesq model has a conventional limit with a finite wavelength, the discrete solution converges to a piece-wise constant function with the discontinuities at lattice points, while the Rosenau model has a nontrivial limit represented by a stationary Young measure (cf. Theil and Levitas, 2000).

\section{Conclusions}

In this paper we studied a simple example of radiative damping in discrete lattices. The goal was to simulate an isolated kink moving uniformly under the action of a given configurational force. We computed an explicit expression for the dissipative function in the discrete model and showed it to be of a dry friction type. We then studied two related quasicontinuous models built on the polynomial (Boussinesq) and rational (Rosenau) approximations of the finite-difference part of the discrete model. Although both models produced excellent agreement with the discrete model at high velocities, they both failed at low velocities where due to lattice resonances the discreteness of the model cannot be ignored. Since the Cauchy problem for the Boussinesq model is ill-posed, preference should be given to the Rosenau and similar higher-order Pade approximations generating much more realistic short wave part of the dispersive spectrum.

One possibility which has not been explored in our paper is that the adequate continuum description of the discrete system, valid in the broad range of wavelengths and wave velocities, is thermodynamical. We recall that the generation of short wave lattice vibrations by the moving defect is in many respects similar to the localized increase of temperature. Moreover, continuum models of phase transitions that take latent heat and heat conductivity into account, generate kinetic relations which are in surprisingly good qualitative agreement with our "first principle" kinetic relation obtained from the discrete model. Specifically in both discrete and nonisothermal models the kinetic curves are nonmonotone and exhibit a finite range of trapping; other similarities include the possibility of supersonic regimes and the multivaluedness of the kinetic functions at sufficiently slow velocities (Ngan and Truskinovsky 1999, 2002). This intriguing similarity is worth pursuing.

\section{Acknowledgements}

This work was supported by the NSF grants DMS-9803572 and DMS-0102841.

\section{Appendix A}

Here we show how solution (11) of the piece-wise linear problem (6)-(10) can be obtained by the Fourier method. We begin by representing the displacement field in the form $u(x)=u_{+}(x)+u_{-}(x)$, where

$$
u_{+}(x)=\left\{\begin{array}{ll}
u(x), & x>0, \\
0, & x<0,
\end{array} \quad u_{-}(x)= \begin{cases}0, & x>0, \\
u(x), & x<0 .\end{cases}\right.
$$


Assume that for $v=k+i s$ the integrals

$$
\hat{u}_{+}(v)=\frac{1}{\sqrt{2 \pi}} \int_{0}^{\infty} u(x) \mathrm{e}^{\mathrm{i} v x} \mathrm{~d} x ; \quad \hat{u}_{-}(v)=\frac{1}{\sqrt{2 \pi}} \int_{-\infty}^{0} u(x) \mathrm{e}^{\mathrm{i} v x} \mathrm{~d} x
$$

exist: the former for $s>0$, the latter for $s<0$. Then for some $s_{+}>0, s_{-}<0$ the Fourier transform can be inverted and we obtain

$$
u(x)=\frac{1}{\sqrt{2 \pi}} \int_{-\infty+\mathrm{i} s_{+}}^{\infty+\mathrm{i} s_{+}} \hat{u}_{+}(v) \mathrm{e}^{-\mathrm{i} v x} \mathrm{~d} v+\frac{1}{\sqrt{2 \pi}} \int_{-\infty+\mathrm{i} s_{-}}^{\infty+\mathrm{i} s_{-}} \hat{u}_{-}(v) \mathrm{e}^{-\mathrm{i} v x} \mathrm{~d} v .
$$

To obtain an expression for $\hat{u}_{+}(v)$, we recall that at $x>0$ the displacement field $u_{+}(x)$ satisfies the equation

$$
v^{2} \frac{\mathrm{d}^{2} u_{+}}{\mathrm{d} x^{2}}-\left[u_{+}(x+1)-2 u_{+}(x)+u_{+}(x-1)\right]+\Omega_{0}^{2} u_{+}(x)=\sigma \Omega_{0}^{2} .
$$

Applying the Fourier transform to Eq. (A.4) and using Eq. (8), we obtain

$$
\begin{aligned}
\hat{u}_{+}(v)= & \frac{1}{\sqrt{2 \pi} L(v)}\left[v^{2} u_{+}^{\prime}(0)-\frac{\mathrm{i} v}{2} v^{2}-\left(\int_{0}^{1} \mathrm{e}^{\mathrm{i} v(x-1)} u(x) \mathrm{d} x\right.\right. \\
& \left.\left.-\int_{-1}^{0} \mathrm{e}^{\mathrm{i} v(x+1)} u(x) \mathrm{d} x\right)+\Omega_{0}^{2} \sigma \int_{0}^{\infty} \mathrm{e}^{\mathrm{i} v x} \mathrm{~d} x\right],
\end{aligned}
$$

where

$$
L(v)=\Omega_{0}^{2}+4 \sin ^{2} \frac{v}{2}-v^{2} v^{2}
$$

Now by using the inverse Fourier transform (A.3) we can write

$$
\begin{aligned}
u_{+}(x)= & \frac{1}{2 \pi} \int_{\mathrm{i} s_{+}-\infty}^{\mathrm{i} s_{+}+\infty} \frac{\mathrm{e}^{-\mathrm{i} v x}}{L(v)}\left[v^{2} u_{+}^{\prime}(0)-\frac{\mathrm{i} v^{2} v}{2}+\sigma \Omega_{0}^{2} \int_{0}^{\infty} \mathrm{e}^{\mathrm{i} v \xi} \mathrm{d} \xi\right. \\
& \left.-\left(\int_{0}^{1} u(\xi) \mathrm{e}^{\mathrm{i} v(\xi-1)} \mathrm{d} \xi-\int_{-1}^{0} u(\xi) \mathrm{e}^{\mathrm{i} v(\xi+1)} \mathrm{d} \xi\right)\right] \mathrm{d} v .
\end{aligned}
$$

One can repeat the same procedure for $u=u_{-}(x)$ and obtain

$$
\begin{aligned}
u(x) & =\Omega_{0}^{2} \frac{\sigma}{2 \pi} \int_{-\infty}^{+\infty} \frac{\mathrm{e}^{\mathrm{i} k x}}{L(k)} \mathrm{d} k \int_{-\infty}^{\infty} \mathrm{e}^{-\mathrm{i} k \xi} \mathrm{d} \xi+\frac{\Omega_{0}^{2}}{2 \pi} \int_{-\infty}^{+\infty} \frac{\mathrm{e}^{\mathrm{i} k x}}{L(k)} \mathrm{d} k \int_{-\infty}^{0} \mathrm{e}^{-\mathrm{i} k \xi} \mathrm{d} \xi \\
& =\sigma+\frac{1}{2}-\frac{\Omega_{0}^{2}}{2 \pi \mathrm{i}} \int_{-\infty}^{+\infty} \frac{\mathrm{e}^{\mathrm{i} k x} \mathrm{~d} k}{k L(k)}
\end{aligned}
$$

In order to select the physically correct solution, we need to interpret the integral in Eq. (A.8) as a contour integral along the path $\gamma$ which does not pass through singular points. Consider first the singularity at $k=0$. By choosing a contour $\gamma$ close to the real 
axis and passing above the point $k=0$ we obtain

$$
u(x)=\sigma-\frac{\Omega_{0}^{2}}{2 \pi \mathrm{i}} \int_{\gamma} \frac{\mathrm{e}^{\mathrm{i} k x}}{k L(k)} \mathrm{d} k .
$$

To compute the integral in Eq. (A.9) we can use the method of residues. For $x>0$ we need to complete the contour $\gamma$ in the upper half-plane and for $x<0$ in the lower half-plane. In this way, we automatically include into the field only the waves which are exponentially damped away from the phase boundary. The real roots of equation $L(k)=0$ require special analysis, because the corresponding waves do not decay at $\pm \infty$. By applying the radiation conditions and computing the residues we obtain (11), (14).

In order to determine the value of applied force $\sigma$ which is necessary to sustain our dynamic displacement profile we need to use the matching condition $u(0)=1 / 2$. From Eq. (A.9) we obtain

$$
\sigma=\frac{1}{2}+\frac{\Omega_{0}^{2}}{2 \pi \mathrm{i}} \int_{\gamma} \frac{\mathrm{d} k}{k L(k)} .
$$

Now by closing the contour of integration in Eq. (A.10) in the upper half-plane, we obtain

$$
\int_{\gamma} \frac{\mathrm{d} k}{k L(k)}=4 \pi \mathrm{i} \sum_{k \in N_{+}(k)} \frac{1}{k L^{\prime}(k)}+2 \pi \mathrm{i} \sum_{L(k)=0, \operatorname{Im} k>0} \frac{A}{A^{2}+B^{2}},
$$

where $N_{+}(k)=\left\{k: L(k)=0 ; \operatorname{Im} k=0 \cup k L^{\prime}(k)>0\right\}$. For the complex roots $k=k_{1}+\mathrm{i} k_{2}$,

$$
\begin{gathered}
A=k_{1} \sin k_{1} \cosh k_{2}-k_{2} \cos k_{1} \sinh k_{2}+\left(k_{2}^{2}-k_{1}^{2}\right) v^{2}, \\
B=k_{2} \sin k_{1} \cosh k_{2}+k_{1} \cos k_{1} \sinh k_{2}-2 k_{1} k_{2} v^{2} .
\end{gathered}
$$

Alternatively, we can compute (A.10) by closing the contour of integration $\gamma$ in the lower half-plane. We obtain

$$
\int_{\gamma} \frac{\mathrm{d} k}{k L(k)}=-2 \pi \mathrm{i}\left[\frac{1}{\Omega_{0}^{2}}+2 \sum_{k \in N_{-}(k)} \frac{1}{k L^{\prime}(k)}+\sum_{L(k)=0, \operatorname{Im} k<0} \frac{A}{A^{2}+B^{2}}\right],
$$

where now $N_{-}(k)=\left\{k: L(k)=0 ; \operatorname{Im} k=0 \cup k L^{\prime}(k)<0\right\}$ and $A$ and $B$ are the same as in Eq. (A.12) due to the symmetry of the complex roots. Since the function $A\left(k_{1}, k_{2}\right)$ is even in both $k_{1}$ and $k_{2}$, and since the complex roots are symmetric with respect to the planes $\operatorname{Im} k=0$ and $\operatorname{Re} k=0$, we obtain

$2 \pi \mathrm{i} \sum_{L(k)=0, \operatorname{Im} k>0} \frac{A}{A^{2}+B^{2}}=-\mathrm{i} \pi\left[\frac{1}{\Omega_{0}^{2}}+2 \sum_{k \in N_{+}(k)} \frac{1}{k L^{\prime}(k)}+2 \sum_{k \in N_{-}(k)} \frac{1}{k L^{\prime}(k)}\right]$. 
By substituting this expression into Eqs. (A.10)-(A.11) we obtain the desired relation for $\sigma$

$$
\sigma=\Omega_{0}^{2} \sum_{k \in N_{ \pm}(k)} \frac{1}{\left|k L^{\prime}(k)\right|}
$$

\section{Appendix B}

In statics our Eq. (2) takes the form

$$
\left(u_{n+1}-2 u_{n}+u_{n-1}\right)-\Omega_{0}^{2}\left(u_{n}-\sigma\right)= \begin{cases}0, & n \geqslant 0, \\ -\Omega_{0}^{2}, & n<0,\end{cases}
$$

where we have already substituted the piece-wise linear force law (3) and used the nondimensional variables (5), (7); the boundary conditions remain the same as in Eq. (10).

Introduce new functions $u_{n}^{+}$and $u_{n}^{-}$, describing the solutions of (B.1) at $n \geqslant 0$ and $n<0$, respectively. The compatibility conditions can be written in the form

$$
u_{0}^{+}=u_{0}^{-} ; \quad u_{-1}^{+}=u_{-1}^{-} .
$$

By solving the finite-difference problem (B.1)-(B.2) we obtain a (stable) solution in the form

$$
u_{n}^{+}=\sigma+\frac{\sqrt{4+\Omega_{0}^{2}}-\Omega_{0}}{2 \sqrt{4+\Omega_{0}^{2}}} r_{2}^{n}, \quad u_{n}^{-}=1+\sigma-\frac{\sqrt{4+\Omega_{0}^{2}}+\Omega_{0}}{2 \sqrt{4+\Omega_{0}^{2}}} r_{1}^{n},
$$

where

$$
r_{1,2}=1+\frac{\Omega_{0}^{2}}{2} \pm \frac{\Omega_{0}}{2} \sqrt{4+\Omega_{0}^{2}} .
$$

Another (unstable) solution representing the so-called Peierls barrier (e.g. Hobart, 1965) can be obtained if instead of conditions (B.2) we consider a different set of matching conditions

$$
u_{0}^{+}=u_{0}^{-}=\frac{1}{2}
$$

Then

$$
u_{n}^{+}=\sigma+\left(\frac{1}{2}-\sigma\right) r_{2}^{n}, \quad u_{n}^{-}=1+\sigma-\left(\frac{1}{2}-\sigma\right) r_{1}^{n},
$$

where $r_{1}$ and $r_{2}$ are the same as in Eq. (B.4). The two solutions satisfy the admissibility conditions for $|\sigma|<\sigma_{\mathrm{p}}^{\mathrm{D}}$ where

$$
\sigma_{\mathrm{p}}^{\mathrm{D}}=\frac{\Omega_{0}}{2 \sqrt{4+\Omega_{0}^{2}}}
$$

is the Peierls force required to start the motion of the kink; at this force the stable and unstable solutions annihilate each other. 
Notice that solution (B.3) and formula (B.7) can also be obtained by direct discrete Fourier transform of Eq. (B.1). Indeed, by transforming Eqs. (B.1) we obtain

$$
\hat{u}^{\mathrm{D}}(k)=\sqrt{2 \pi} \sigma \delta(k)+\sqrt{\frac{\pi}{2}} \delta(k)-\frac{1}{\sqrt{2 \pi}} \frac{\Omega_{0}^{2}}{\left(1-\mathrm{e}^{-\mathrm{i} k}\right) L_{0}(k)},
$$

where

$$
\hat{u}^{\mathrm{D}}(k)=\frac{1}{\sqrt{2 \pi}} \sum_{n=-\infty}^{n=\infty} u_{n} \mathrm{e}^{-\mathrm{i} k n}
$$

and

$$
L_{0}(k)=\Omega_{0}^{2}+4 \sin ^{2} \frac{k}{2}
$$

Now if we make an inverse discrete Fourier transform of $\hat{u}^{\mathrm{D}}(k)$

$$
u_{n}=\frac{1}{\sqrt{2 \pi}} \int_{-\pi}^{+\pi} \hat{u}^{\mathrm{D}}(k) \mathrm{e}^{\mathrm{i} k n} \mathrm{~d} k,
$$

we obtain

$$
u_{n}=\sigma+\frac{1}{2}-\frac{\Omega_{0}^{2}}{2 \pi} \int_{-\pi}^{\pi} \frac{\mathrm{e}^{\mathrm{i} k n}}{\left(1-\mathrm{e}^{-\mathrm{i} k}\right) L_{0}(k)} \mathrm{d} k .
$$

The next step is to extend the above solution analytically by zero from the interval $-\pi<k<\pi$ to the whole real axis and choose the contour $\gamma$ passing above the pole at the origin. Then we can write

$$
u_{n}=\sigma+\frac{\Omega_{0}^{2}}{2 \pi} \int_{\gamma} \frac{\mathrm{e}^{\mathrm{i} k n}}{L_{0}(k)\left(\mathrm{e}^{-\mathrm{i} k}-1\right)} \mathrm{d} k .
$$

Since equation $L_{0}(k)=0$ does not have real roots, we can compute the integral in Eq. (B.13) by closing the contour in the upper half-plane for $n>0$, and in the lower half-plane for $n<0$. The relevant complex roots of the equation $L_{0}(k)=0$ are: $p_{1}=2 \mathrm{i} \sinh ^{-1}\left(\Omega_{0} / 2\right)$ in the upper half-plane, and $p_{2}=-2 \mathrm{i} \sinh ^{-1}\left(\Omega_{0} / 2\right)$ in the lower half-plane. We obtain

$$
\begin{aligned}
& u_{n}^{+}=\sigma+\left.\mathrm{i} \frac{\Omega_{0}^{2} \mathrm{e}^{\mathrm{i} k n}}{L_{0}^{\prime}(k)\left(\mathrm{e}^{-\mathrm{i} k}-1\right)}\right|_{k=p_{1}}, \\
& u_{n}^{-}=\sigma+1-\left.\mathrm{i} \frac{\Omega_{0}^{2} \mathrm{e}^{\mathrm{i} k n}}{L_{0}^{\prime}(k)\left(\mathrm{e}^{-\mathrm{i} k}-1\right)}\right|_{k=p_{2}} .
\end{aligned}
$$

Now by noticing that $\mathrm{e}^{\mathrm{i} p_{1} n}=r_{2}^{n}$ and $\mathrm{e}^{\mathrm{i} p_{2} n}=r_{1}^{n}$ we can rewrite (B.14) in form (B.3), and from condition (B.5) obtain the expression for Peierls force (B.7).

Now for consistency of our construction we need to show that the static solution (B.3), (B.12) is compatible with the dynamic solution discussed in the main body of the paper. The idea of the analysis below can be traced to the works of Celli and Flytzanis (1970) and Slepyan (1982). First notice that we need to identify the limiting dynamic solution $u(x)$ represented by a function of continuum argument with the static solution $u_{n}$ represented by a function of a discrete argument. Suppose, that at $v=0$ 
there exists a piece-wise constant function $u_{0}(x)$ describing discrete solution of the static problem, which is the limit of the solution of the dynamic problem $u(x-v t)$ at $v=0$. By computing the Fourier transform of the function $u_{0}(x)$ we can write

$$
\begin{aligned}
\hat{u}_{0}(k) & =\frac{1}{\sqrt{2 \pi}} \int_{-\infty}^{\infty} u_{0}(\eta) \mathrm{e}^{-\mathrm{i} k \eta} \mathrm{d} \eta \\
& =\frac{1}{\sqrt{2 \pi}} \sum_{n=-\infty}^{n=\infty} \int_{n}^{n+1} u_{0}(x) \mathrm{e}^{-\mathrm{i} k x} \mathrm{~d} x \\
& =\frac{1}{\sqrt{2 \pi}} \sum_{n=-\infty}^{n=\infty} u_{n} \frac{\mathrm{e}^{-\mathrm{i} k(n+1)}-\mathrm{e}^{-\mathrm{i} k n}}{-\mathrm{i} k} \\
& =\frac{1-\mathrm{e}^{-\mathrm{i} k}}{\mathrm{i} k} \hat{u}^{\mathrm{D}}(k) .
\end{aligned}
$$

Then by noticing that in the limit $v \rightarrow 0$ the Fourier image of dynamic solution is

$$
\hat{u}_{0}(k)=\sqrt{2 \pi} \sigma \delta(k)+\sqrt{\frac{\pi}{2}} \delta(k)-\frac{\Omega_{0}^{2}}{\sqrt{2 \pi} \mathrm{i} k L_{0}(k)}
$$

and by applying to (B.16) the rescaling procedure (B.15), we obtain

$$
\lim _{v \rightarrow 0} u(n-v t)=u_{n}=\sigma+\frac{1}{2}+\frac{\Omega_{0}^{2}}{2 \pi} \int_{-\pi}^{+\pi} \frac{\mathrm{e}^{\mathrm{i} k n}}{L_{0}(k)\left(\mathrm{e}^{-\mathrm{i} k}-1\right)} \mathrm{d} k,
$$

which is exactly (B.12).

In the Boussinesq approximation instead of (B.1) we have

$$
u_{x x}+\frac{u_{x x x x}}{12}-\Omega_{0}^{2}(u-\sigma)= \begin{cases}0, & u<\frac{1}{2}, \\ -\Omega_{0}^{2}, & \frac{1}{2}<u .\end{cases}
$$

The boundary and compatibility conditions are the same as in the dynamic case. The compatibility conditions at $x=0$ take the form

$$
[u]=\left[u_{x}\right]=\left[u_{x x}\right]=\left[u_{x x x}\right]=0 .
$$

The solution of (B.18), (B.19) can be written explicitly

$$
u(x)=\left\{\begin{array}{l}
\frac{1}{4}\left(1+\sqrt{\frac{3}{3+\Omega_{0}^{2}}}\right) \mathrm{e}^{-r_{1} x}+\left(\frac{1}{4}\left(1-\sqrt{\frac{3}{3+\Omega_{0}^{2}}}\right)-\sigma\right) \cos r_{2} x+\sigma, \quad x>0, \\
-\frac{1}{4}\left(1+\sqrt{\frac{3}{3+\Omega_{0}^{2}}}\right) \mathrm{e}^{r_{1} x}+\left(\frac{1}{4}\left(\sqrt{\frac{3}{3+\Omega_{0}^{2}}}-1\right)-\sigma\right) \cos r_{2} x+\sigma+1, x<0,
\end{array}\right.
$$

where

$$
r_{1,2}=\sqrt{\mp 6+6 \sqrt{1+\frac{\Omega_{0}^{2}}{3}}}
$$


From the admissibility conditions we find that $|\sigma|<\sigma_{\mathrm{p}}^{\mathrm{B}}$. Here

$$
\sigma_{\mathrm{p}}^{\mathrm{B}}=\frac{1}{4}\left(1-\sqrt{\frac{3}{3+\Omega_{0}^{2}}}\right)
$$

is the desired expression for the Peierls force.

Finally in the continuum case we obtain

$$
u_{x x}-\Omega_{0}^{2} u= \begin{cases}-\Omega_{0}^{2} \sigma, & n \geqslant 0, \\ -\Omega_{0}^{2}-\Omega_{0}^{2} \sigma, & n<0 .\end{cases}
$$

The solution of the above equation satisfying the matching conditions $[u]=\left[u_{x}\right]=0$ at $x=0$ is

$$
u(x)= \begin{cases}\frac{1}{2} \mathrm{e}^{-\Omega_{0} x}+\sigma, & x \geqslant 0, \\ -\frac{1}{2} \mathrm{e}^{\Omega_{0} x}+\sigma+1, & x<0 .\end{cases}
$$

Since it satisfies admissibility conditions only for $\sigma=\sigma_{\mathrm{p}}^{\mathrm{C}}=0$, the corresponding Peierls force is equal to zero.

\section{References}

Abeyaratne, R., Vedantam, S., 1999. Propagation of a front by kink motion. In: Argoul, P, et al. (Ed.), IUTAM Symposium on Variations of Domains and Free Boundary Problems in Solid Mechanics, Kluwer, Dordrecht, pp. 77-84.

Al'shitz, V.A., Indenbom, V.L., 1975. Dynamic dragging of dislocations. Sov. Phys.-Usp. 18 (1), 1-20.

Atkinson, W., Cabrera, N., 1965. Motion of a Frenkel-Kontorova dislocation in a one-dimensional crystal. Phys. Rev. A 138 (3), A763-A766.

Boussinesq, M.J., 1872. Theorie des ondes etde remous qui se propagent le longd'un canal rectangulaire horizontal, en communiquant au liquide contenu dans cecanal des vitesses sensiblement parailles de la surface au fond. J. Math. Pures. Appl. (Ser. 2) 17, 55-108.

Braun, O., Kivshar, Y., 1998. Nonlinear dynamics of the Frenkel-Kontorova model. Phys. Rep. 306, 1-108.

Cahn, J.W., Mallet-Paret, J., Van Vleck, E.S., 1999. Traveling wave solutions for systems of ODEs on a two-dimensional spatial lattice. SIAM J. Appl. Math. 59 (2), 455-493.

Celli, V., Flytzanis, N., 1970. Motion of a screw dislocation in a crystal. J. Appl. Phys. 41 (11), 4443-4447.

Christov, C.I., Maugin, G.A., Velarde, M.E., 1996. Well-posed Boussinesq paradigm with purely spatial higher-order derivatives. Phys. Rev. E 54, 3621-3638.

Frenkel, J., Kontorova, T., 1938. On the theory of plastic deformation and twinning. Proc. Z. Sowj. 13, $1-10$.

Hobart, R., 1965. Peierls stress dependence on dislocation width. Peierls-Barrier minima. J. Appl. Phys. 36 (6), 1944-1952.

Kunin, I.A., 1982. Elastic Media with Microstructure. 1. One-dimensional Models. Springer, New York.

Marder, M., Gross, S., 1995. Origin of crack tip instabilities. J. Mech. Phys. Solids 43 (1), 1-48.

Ngan, S.C., Truskinovsky, L., 1999. Thermal trapping and kinetics of martensitic phase boundaries. J. Mech. Phys. Solids 47 (1), 141-172.

Ngan, S.C., Truskinovsky, L., 2002. Thermoelastic aspects of nucleation in solids. J. Mech. Phys. Solids 50, 1193-1229.

Pego, R.L., Smereka, P., Weinstein, M.I., 1995. Oscillatory instability of solitary waves in a continuum model of lattice vibrations. Nonlinearity 8, 921-941.

Rosenau, P., 1986. Dynamics of nonlinear mass spring chains near the continuum limit. Phys. Rev. Lett. A $118(5), 222-227$. 
Slepyan, L.I., 1981. Dynamics of a crack in a lattice. Sov. Phys.-Dokl. 26 (5), 538-540.

Slepyan, L.I., 1982. The relation between the solutions of mixed dynamical problems for a continuous elastic medium and a lattice. Sov. Phys.-Dokl. 27 (9), 771-772.

Slepyan, L.I., 2000. Dynamic factor in impact, phase transition and fracture. J. Mech. Phys. Solids 48, 927-960.

Slepyan, L.I., 2001a. Feeding and dissipative waves in fracture and phase transition. 1. Some 1D structures and a square-cell lattice. J. Mech. Phys. Solids 49, 469-511.

Slepyan, L.I., 2001b. Feeding and dissipative waves in fracture and phase transition. 2. Phase-transition waves. J. Mech. Phys. Solids 49, 513-550.

Slepyan, L.I., 2001c. Feeding and dissipative waves in fracture and phase transition. 3. Triangular-cell lattice. J. Mech. Phys. Solids 49, 2839-2875.

Slepyan, L.I., Troyankina, L.V., 1984. Fracture wave in a chain structure. J. Appl. Mech. Techn. Phys. 25 (6), 921-927.

Theil, F., Levitas, V.I., 2000. A study of a Hamiltonian model for martensitic phase transformations including micro-kinetic energy. Math. Mech. Solids 5 (3), 337-368.

Wattis, J., 1993. Approximations to solitary waves on lattices: 2. Quasi-continuum methods for fast and slow waves. J. Phys. A 26, 1193-1209. 